\title{
Pacific
}

Journal of

Mathematics

\section{METRIC PROPERTIES OF HIGHER-DIMENSIONAL THOMPSON'S GROUPS}

\author{
José BuRillo AND SEAN Cleary
}




\title{
METRIC PROPERTIES OF HIGHER-DIMENSIONAL THOMPSON'S GROUPS
}

\author{
José Burillo And SEAn Cleary
}

\begin{abstract}
Higher-dimensional Thompson's groups $n \boldsymbol{V}$ are finitely presented groups that generalize dyadic self-maps of the unit interval to dyadic self-maps of $n$-dimensional unit cubes. We describe some of the metric properties of these groups. We describe their elements based upon tree-pair diagrams and give upper and lower bounds for word length in terms of the size of the diagrams. Though these bounds are somewhat separated, we show that there are elements realizing the lower bounds and that the fraction of elements that are close to the upper bound converges to 1 , showing that the bounds are optimal and that the upper bound is generically achieved.
\end{abstract}

\section{Introduction}

Thompson's groups provide a wide range of interesting examples of unusual grouptheoretic behavior. The family of Thompson's groups includes the original groups described by Thompson, commonly denoted $F, T$ and $V$, as well as generalizations in many different directions. The bulk of these generalizations includes groups that can be regarded as self-maps of the unit interval. Brin [2004; 2005] generalized $V$ to higher-dimensional groups $n V$, which are described naturally in terms of dyadic self-maps of $n$-dimensional cubes. Little is known about these groups aside from their simplicity. Brin describes their elements geometrically in terms of dyadic interpolations of collections of dyadic blocks, and gives presentations for $2 \mathrm{~V}$.

We describe elements in higher-dimensional Thompson's groups as being given by tree-pair diagrams. Though these diagrams usually take advantage of the natural left-to-right ordering of subintervals of the unit interval, by using several types of carets, Brin describes elements of $n V$ via tree-pair diagrams. A natural question is how the size of these tree-pair diagrams corresponds to the word length of elements with respect to finite generating sets.

MSC2000: 20F65.

Keywords: Thompson's groups.

The authors are grateful for the hospitality of the Centre de Recerca Matemàtica. Burillo acknowledges support from MEC grant MTM2006-13544-C02-02 and is grateful for the hospitality of The City College of New York. This work was supported in part by NSF grant 0811002 and grants from The City University of New York PSC-CUNY Research Award Program. 
We give upper and lower bounds for the word lengths of elements of higherdimensional Thompson's groups $n V$ with respect to finite generating sets, in terms of the size of tree-pair descriptions of elements. An element with a minimal treepair description of size $N$ has word length between $\log N$ and $N \log N$ (up to the standard affine equivalences) with respect to the standard finite generating set. This of course also thus holds for any finite generating set.

\section{Background on higher-dimensional Thompson's groups}

Brin [2004] describes higher-dimensional Thompson's groups $n V$, giving presentations and showing that the group $2 V$ is simple. Brin [2004; 2005] defines $n V$ as a subgroup of the homeomorphism group of the $n$-fold product of the Cantor set with itself, and then shows there are a number of equivalent characterizations. The one we use here is Brin's characterization [2005] in terms of equivalence classes of labeled tree pair diagrams. The properties of the groups $n V$ relevant here are as follows.

We denote the unit interval $[0,1]$ by $I$, and the $n$-dimensional unit cube by $I^{n}$.

An element of $V$ is given by two finite-rooted binary trees with the same number of leaves and a permutation, which represents a bijection between the leaves of the two trees. Hence an element of $V$ can be seen as a triple $\left(T_{+}, \pi, T_{-}\right)$, where $T_{+}$ and $T_{-}$are trees with $k$ leaves, and $\pi \in \mathscr{T}_{k}$.

Each binary tree can be seen as a way of subdividing the interval $I$ into dyadic subintervals of the type $\left[i / 2^{r}, i+1 / 2^{r}\right]$, where $r>0$ and $0 \leq i<2^{r}$. A rooted binary tree gives instructions for successive halvings of subintervals to obtain a particular dyadic subdivision. Given two binary trees with $n$ leaves and a permutation in $\mathscr{Y}_{n}$, an element in $V$ is represented as a left-continuous map of the interval into itself, sending each interval in the first subdivision to a corresponding interval in the second subdivision, as specified by the given permutation. Though not every dyadic subdivision of $I$ can be obtained by a successive halving process described by trees, every dyadic subdivision has a refinement that can be obtained by a successive halving process.

For more details on the group $V$, including presentations and a proof of its simplicity, see Cannon, Floyd and Parry [1996] as well as Brin [2004].

To obtain an element in $2 \mathrm{~V}$, we will define a partition of $I^{2}$ into dyadic rectangles of the type

$$
\left[\frac{i}{2^{r}}, \frac{i+1}{2^{r}}\right] \times\left[\frac{j}{2^{s}}, \frac{j+1}{2^{s}}\right] .
$$

Though again, not every such partition can be realized by successive halving processes, every such partition has a refinement that can be realized in that way. For the two-dimensional successive halving process, there are two possible halvings 
that can occur at each stage to a specified dyadic rectangle - a horizontal or a vertical subdivision. We can obtain a refinement of any dyadic partition of $I^{2}$ via iterated horizontal and vertical subdivisions. This gives a natural means of describing elements of $2 V$ as pairs of dyadic subdivisions, each with $m$ rectangles, and a permutation in $S_{m}$ giving the bijection between the rectangles.

We can describe elements of $2 \mathrm{~V}$ also with pairs of binary trees, with the nodes representing steps in successive halving processes to represent the refined subdivisions. Since there are two types of subdivisions, vertical and horizontal, we will consider binary trees that contain two types of carets, vertical and horizontal carets, represented by triangular and square carets, respectively.

As with other tree-based definitions of Thompson's groups, there are numerous representatives of a given group element, with natural equivalence relations coming from expansions and contractions of leaves of trees. To multiply two elements, we typically find representatives for each of them where the trees are compatible for a natural multiplication via composition.

We define the group $2 V$ as the set of equivalence classes of triples $\left(T_{+}, \pi, T_{-}\right)$ in which the trees are labeled to indicate the successive halving process and $\pi$ is a permutation between the leaves of the trees (where each leaf represents a dyadic rectangle).

To fix a labeling convention, we will represent a vertical subdivision with a traditional triangular caret, where the left and right leaves naturally represent the left and right rectangles. A horizontal subdivision will be represented by a square caret; in it, the left leaf represents the bottom rectangle, and the right leaf represents the top rectangle, as shown below.

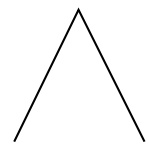

(1)

(2)

vertical caret and subdivision

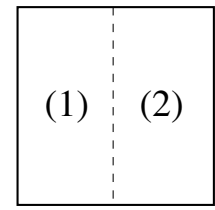

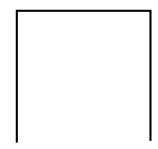

(1)

(2)

horizontal caret and subdivision

(2)

(1)

We say a representative of an element is reduced if for each caret $c$ in $T_{-}$of with two leaves, the permutation $\pi$ does not map both leaves of $c$ to leaves of the same caret of the same type in $T_{+}$in the same order. We say a representative 

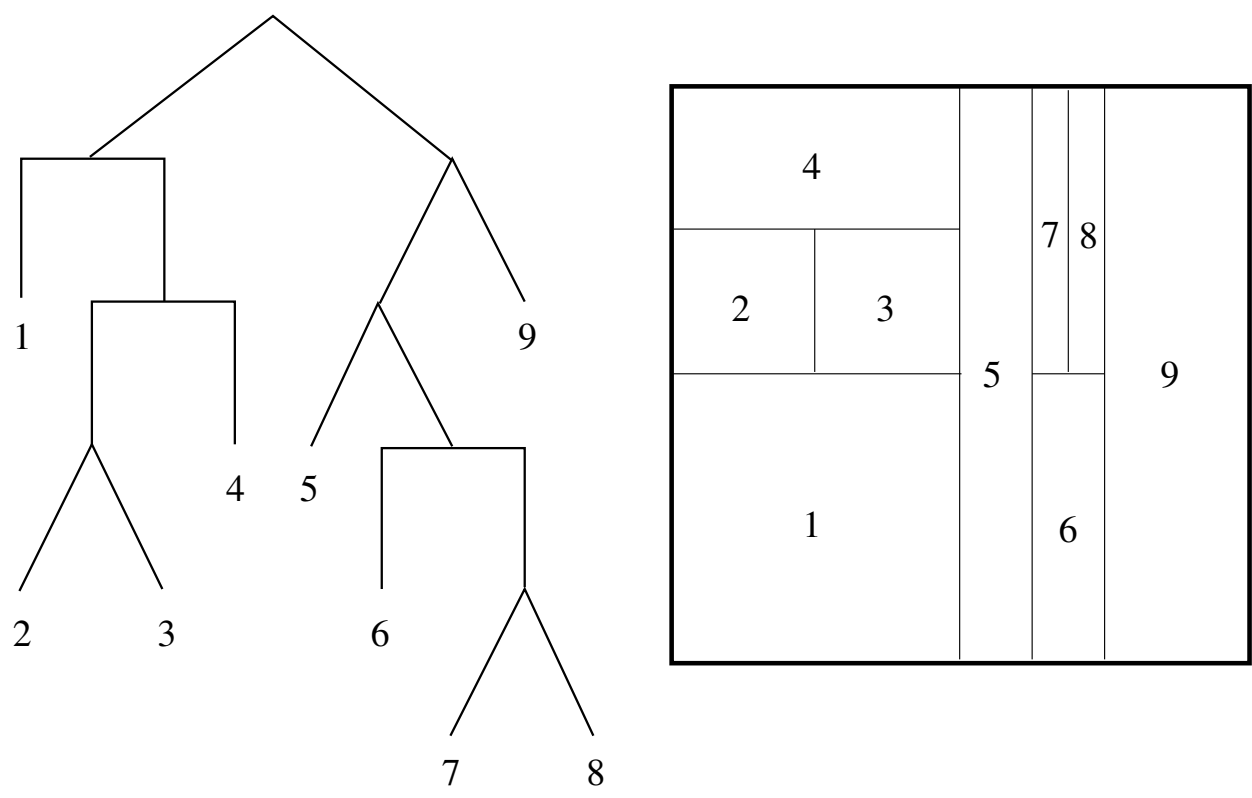

Figure 1. An example of a binary tree with the two types of carets, and its corresponding partition of $I^{2}$.

of an element is of minimal size if it has the minimal number of carets among all representatives in its equivalence class, and we denote by $N(x)$ the number of carets in a minimal size representative of an element $x$. Unlike the case of $V$, but similar to that of $F(n, m)$ (see Wladis [2007]), each element of minimal size may have multiple distinct representatives.

See Figure 1 for an example of a partition of $I^{2}$ and its corresponding binary tree. Every dyadic partition of the unit square has a refinement that can be obtained with a binary tree with these two types of carets.

For the general groups $n V$, the partitions are divisions of the unit $n$-cube in $n$ rectangles of dyadic lengths, and the corresponding binary trees have $n$ types of carets. For simplicity, we state our results for $n=2$, but all of the results below extend naturally to $n>2$.

The fact that we have vertical and horizontal subdivisions brings new relations to the group. These relations arise when both types of subdivisions are combined in different orders to obtain different descriptions of the same dyadic partition of $I^{2}$. The obvious relation (and the one from which all other relations are deducible) is the combination of one subdivision of each type in the two possible orders, as illustrated in Figure 2.

Subdividing in both the vertical and horizontal directions once, but in the two possible orders, gives the same dyadic partitions, but according to our convention 

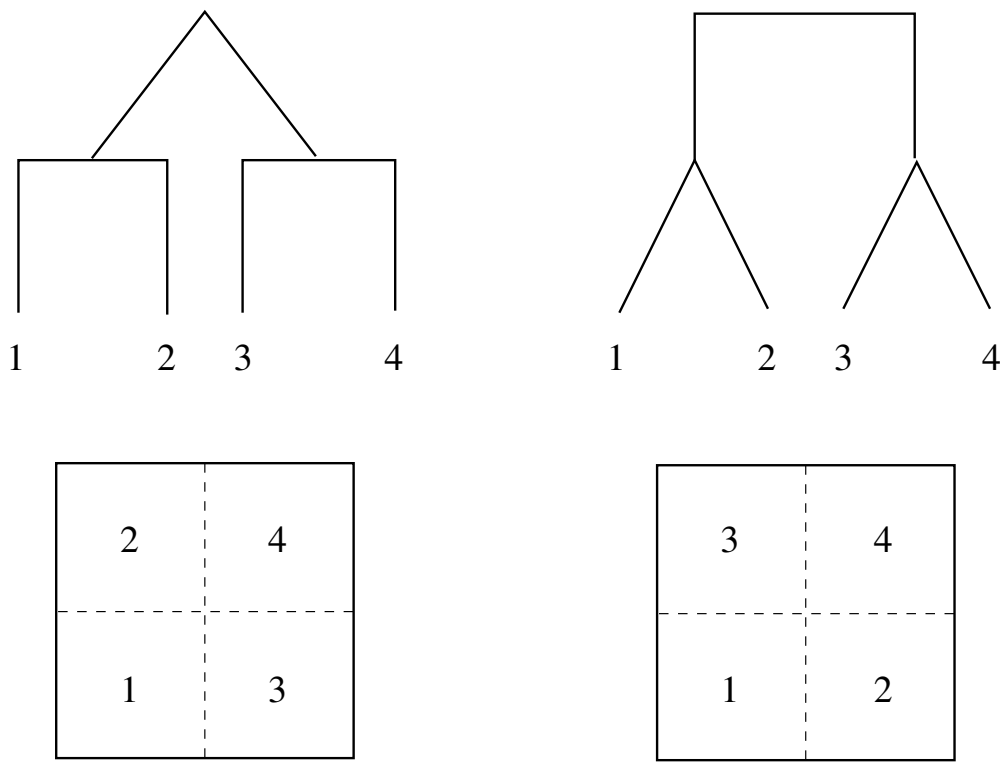

4

23

4

Figure 2. The relation obtained when performing vertical and horizontal subdivisions.

the resulting rectangles are numbered in different way. This makes using tree diagrams to express the multiplication of elements tricky and quite unwieldy for large elements. Even though the leaves of the two-caret-type tree diagrams are ordered in a natural way, this order is not apparent in the square, and it is not preserved when different diagrams represent the same partition. Thus, there are (nonminimal) diagrams representing the identity whose leaves are ordered in different ways an example is illustrated in Figure 3.
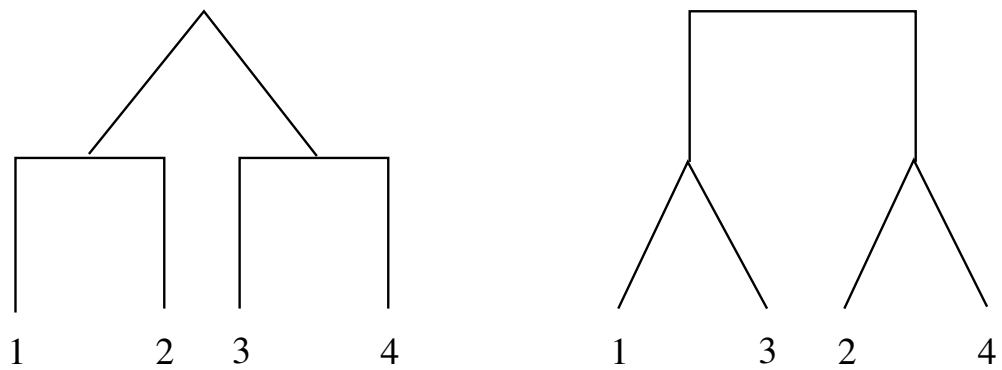

Figure 3. A tree-pair diagram for the identity element that has a nonidentity permutation. 


\section{Families of generators}

Brin [2004; 2005] showed that the groups $n V$ are finitely generated and gave several generating sets for $2 \mathrm{~V}$. As is common with groups of the Thompson family, there is an infinite presentation, which is useful for its symmetry and regularity and which has a finite subpresentation. For the purposes of word length, we work with a standard finite generating set as described by Brin. We next define the families of generators for $2 \mathrm{~V}$, following the usual procedure in Thompson's groups; see Cannon, Floyd and Parry [1996]. We build the generators on a backbone of an all-right tree with only vertical carets.

(1) The generators $A_{n}$ involve only vertical subdivisions, and they are the traditional generators of Thompson's group $F$.

(2) The generators $B_{n}$ have the one nonright caret replaced by a horizontal one.

(3) The generators $C_{n}$ have the last caret of the all-right tree of horizontal type, and they are used to build horizontal carets on the right-hand-side of the tree, as will be seen later.

(4) The generators $\pi_{n}$ and $\bar{\pi}_{n}$ are permutations built on an all-right tree with only vertical carets. These generators are exactly equal to those for the subgroup $V$ appearing as the purely vertical elements.

Theorem 2.1 [Brin 2004; 2005]. The families $A_{n}, B_{n}, C_{n}, \pi_{n}$ and $\bar{\pi}_{n}$ generate the group $2 V$.

Proof. Since our proof is quite different from Brin's, we will include it here and use aspects of it later for metric considerations.

An element of $2 V$ is given by a triple $\left(T_{+}, \pi, T_{-}\right)$, where the two trees are composed of the two types of carets. First, we subdivide a given element into three elements: $\left(T_{+}, \mathrm{id}, R_{k}\right),\left(R_{k}, \pi, R_{k}\right)$, and $\left(R_{k}, \mathrm{id}, T_{-}\right)$, where $R_{k}$ is the allright tree with $k$ leaves and only vertical carets. Clearly $\left(R_{k}, \pi, R_{k}\right)$ is product of the permutation generators, as is the case in $V$ already.

To obtain the element $\left(T_{+}\right.$, id, $\left.R_{k}\right)$, we will concentrate first on the backbone of the tree $T_{+}$, that is, the sequence of carets in the right hand side. If this sequence of carets has horizontal carets in the positions $m_{1}, m_{2}, \ldots, m_{p}$, then the product of the generators $C_{m_{1}} C_{m_{2}} \cdots C_{m_{p}}$ produces exactly a backbone with horizontal carets in the desired positions. We denote this backbone by $K$; that is, $K$ is the subtree of $T_{+}$that consists only of right carets. Thus, at this stage, we have constructed the element $\left(K\right.$, id, $\left.R_{m_{p}}\right)$.

Once the backbone is constructed, each new caret is obtained by a generator of the type $A_{i}$ or $B_{i}$. To attach a vertical caret from the leaf labeled $i$, we only need to multiply by $A_{i}$ on the right. Similarly, to attach a horizontal caret to leaf $i$, we 


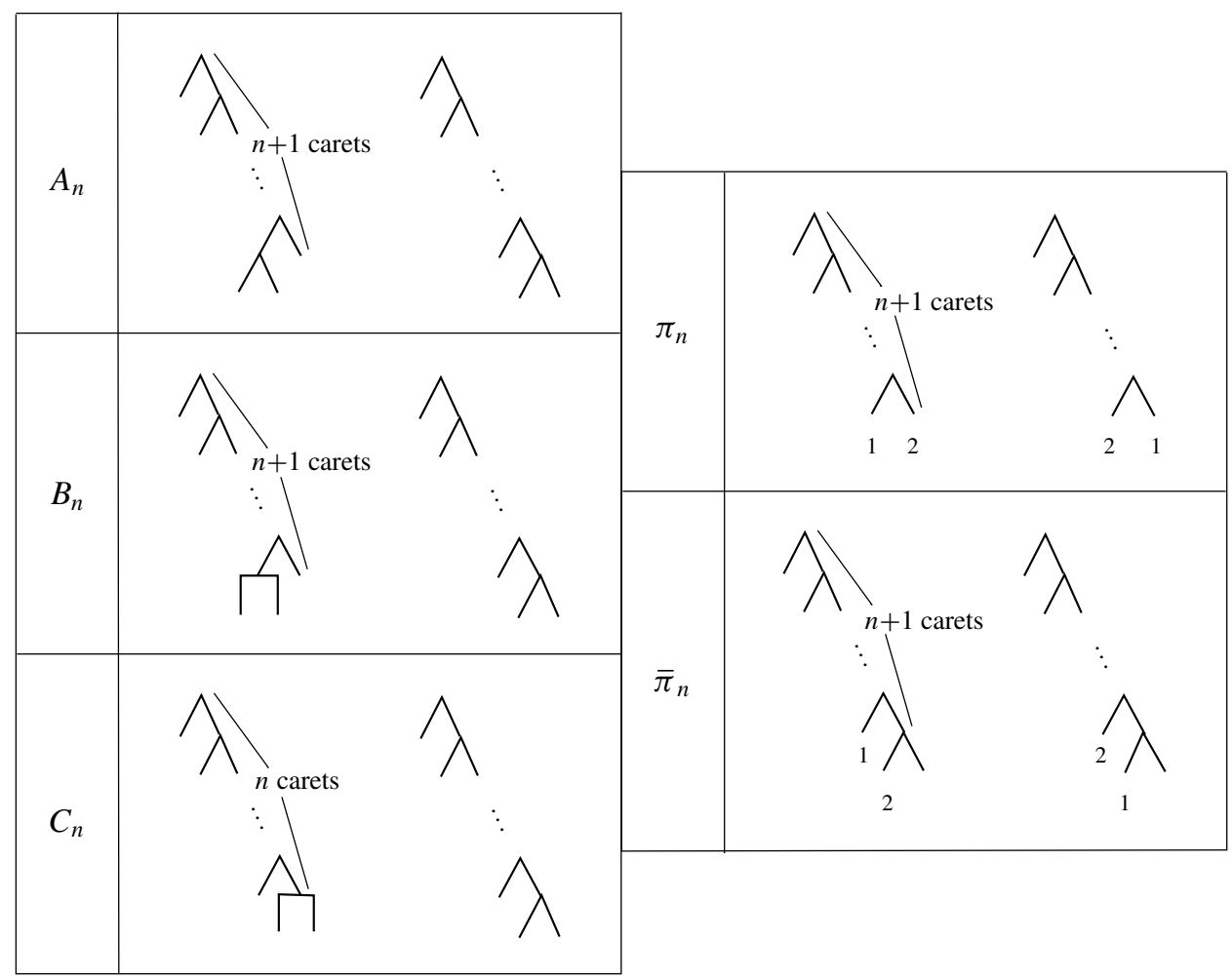

Figure 4. Generators of $2 V$.

multiply by $B_{i}$ on the right. This multiplication process is shown in Figure 5, with an example illustrated in Figure 6.

This proves that the element $\left(T_{+}, \mathrm{id}, R_{k}\right)$ is product of the generators $A_{i}, B_{i}$ and $C_{i}$, and using inverses, we see that the entire group is generated by the full family of generators.

An element of the type $\left(T_{+}\right.$, id, $\left.R_{k}\right)$ is called a positive element of $2 V$. Positive elements can always be written as products of the generators $A_{i}, B_{i}$ and $C_{i}$ without using their inverses.

In the process of proving Brin's theorem, we have obtained this:

Theorem 2.2. Elements of $2 \mathrm{~V}$, with respect to the standard infinite generating set $\left\{A_{i}, B_{i}, C_{i}, \pi_{i}, \bar{\pi}_{n}\right\}$, have these properties:

- Any positive element always admits an expression of the type

$$
C_{m_{1}} \cdots C_{m_{p}} W_{1}\left(A_{i_{1}}, B_{i_{1}}\right) \cdots W_{r}\left(A_{i_{r}}, B_{i_{r}}\right) .
$$



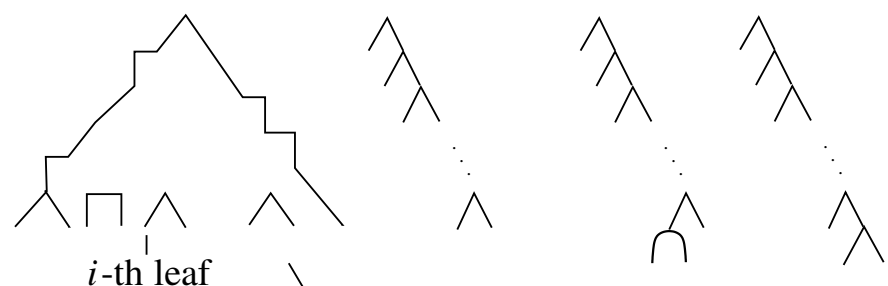

$A_{i}$ or $B_{i}$
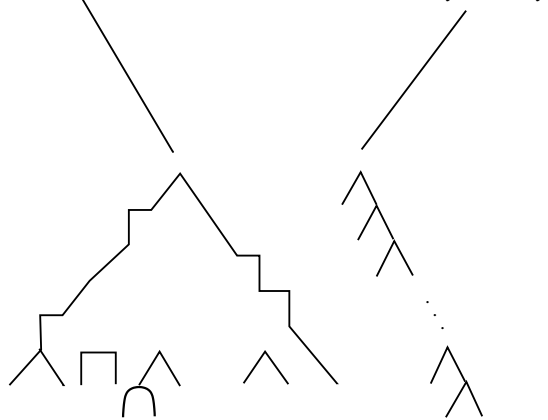

Figure 5. The building process: attaching a caret to the $i$-th leaf by multiplying by $A_{i}$ or $B_{i}$.

where the $W_{i}$ are words on the positive generators $A_{i}$ and $B_{i}$ and never their inverses, and where $m_{1}<m_{2}<\cdots<m_{p}$ and $i_{1}<i_{2}<\cdots<i_{r}$.

- Each element admits an expression $P \Pi Q^{-1}$, where $P$ and $Q$ are positive elements and $\Pi$ is a permutation on an all-right vertical tree, and thus a word in the $\pi_{i}$ and $\bar{\pi}_{i}$.

This expression will be used as a seminormal form for elements of $2 \mathrm{~V}$.

Brin also shows that several finite generating sets suffice to generate each $n V$. We will use the finite generating set $\left\{A_{0}, A_{1}, B_{0}, B_{1}, C_{0}, C_{1}, \pi_{0}, \pi_{1}, \bar{\pi}_{0}, \bar{\pi}_{1}\right\}$. This set is larger than the smallest ones used by Brin, but is more convenient for our methods below.

\section{Metric properties}

We will be interested in metric properties up to the standard affine equivalence, defined here.

Definition 3.1. Given two functions $f, g: G \rightarrow \mathbb{R}$, we say that $f \prec g$ if there exists a constant $C>0$ such that $f(x) \leq C g(x)$ for all $x \in G$. We say that $f$ and $g$ are equivalent, written $f \sim g$, if $f \prec g$ and $g \prec f$.

Elements of Thompson's groups admit representations as diagrams with binary trees in one form or another, perhaps with associated permutations or braids between the leaves. For several of these groups, the distance $|x|$ from an element $x$ 

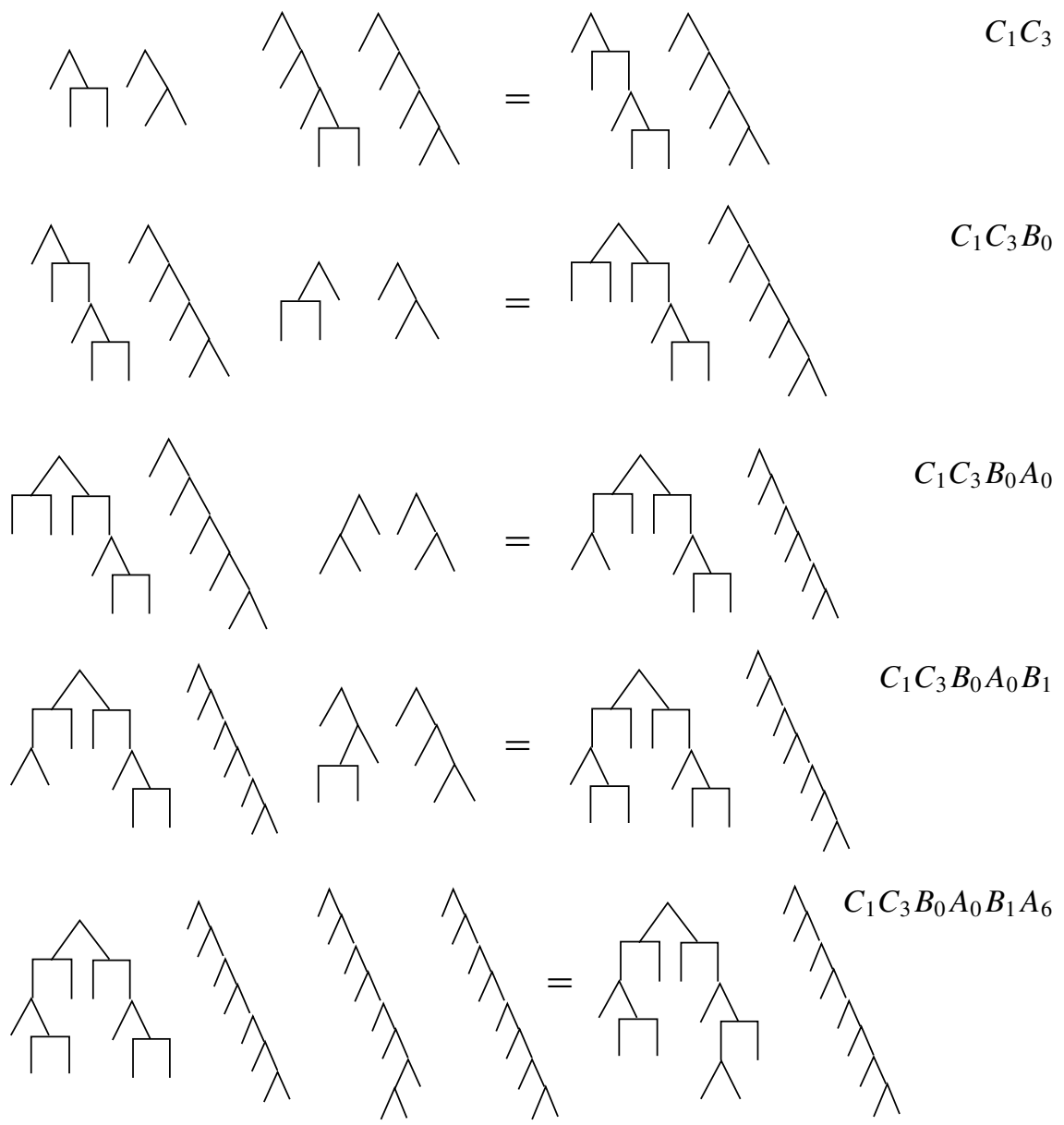

Figure 6. Constructing an element generator-by-generator.

to the identity is closely related with the number of carets $N(x)$ of the minimal diagram, indicating that the complexity of the minimal (reduced) diagram is a good indication of the size of the element.

In Thompson's groups $F$ and $T$, the two functions are equivalent: for both groups we have $|x| \sim N(x)$; see Burillo, Cleary and Stein [2001] and Burillo, Cleary, Stein and Taback [2009]. This result cannot hold in $V$, for counting reasons. The number of elements of $V$ whose trees have $N$ carets is at least the order of $N$ ! (due to the permutations), while the number of distinct elements of length $N$ can be at most exponential in $N$ in any group.

The best possible bound for the number of carets in terms of word length in the group $V$ is an inequality proved by Birget as [2004, Theorem 3.8], which says that 
$N(x) \prec|x| \prec N(x) \log N(x)$ and that these bounds are optimal, in a sense similar to that which will be made precise in Section 4.

In the case of $V$, the lower bounds on word length are linear in the number of carets. Therefore, the standard inclusions $F \subset T \subset V$ are all quasiisometric embeddings; that is, $F$ is undistorted in $T$, and $T$ is undistorted in $V$. This property will be no longer true for inclusions in $2 \mathrm{~V}$, as we will show in Section 5.

In this section we will prove the analog for $2 V$ of the inequality above. In the next section, we address optimality of the bounds.

Theorem 3.2. In the group $2 V$, the word length of an element $|x|$ and number of carets $N(x)$ in a minimal size tree-pair representative satisfy

$$
\log N(x) \prec|x| \prec N(x) \log N(x),
$$

and the bounds cannot be improved.

Proof. We defer the proof of its optimality to the following section.

The upper bound is proved the standard way. We take a positive word $P$, and according to Theorem 2.2, write it as

$$
C_{m_{1}} \cdots C_{m_{p}} W_{1}\left(A_{i_{1}}, B_{i_{1}}\right) \cdots W_{r}\left(A_{i_{r}}, B_{i_{r}}\right) .
$$

Then, we rewrite each generator using the relations

$$
A_{i+1}=A_{0}^{-i+1} A_{1} A_{0}^{i-1}, \quad B_{i+1}=A_{0}^{-i+1} B_{1} A_{0}^{i-1}, \quad C_{i+1}=A_{0}^{-i+1} C_{1} A_{0}^{i-1}
$$

for all $i>1$. Since the conjugating element is always $A_{0}$, cancellations ensure that the length stays approximately the same. The bound $N \log N$ appears because of the permutations, since $N \log N$ is the diameter of $\mathscr{Y}_{N}$ with respect to the relevant transpositions.

For the lower bound, we note that if an element has $k$ carets, when it is multiplied by a generator it is possible that the multiplication could have up to $4 k$ carets. If an element has, for instance, only horizontal subdivisions, when multiplied by $A_{1}$ the number of subdivisions (and hence the number of carets) becomes $4 k$, since each one of the previous subdivision is divided in four, as illustrated in Figure 7.

If each multiplication by a generator can multiply the number of carets by 4 , an element of length $\ell$ could have up to $4^{\ell}$ carets. Other generators may increase word length by additive factors, but the worst case is exactly the increase by a factor of 4 . Hence $N(x) \leq 4^{\ell}$, so $\log N(x) \leq|x|$.

\section{Optimality of bounds}

To show the optimality of the bounds in Theorem 3.2, we first describe some properties of the lower bound for word length in terms of the number of carets. 

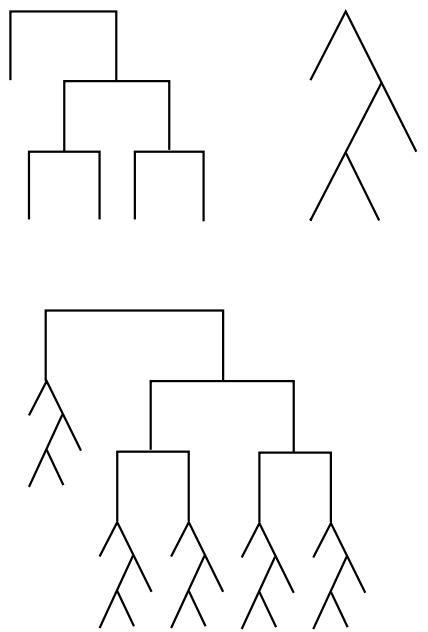

Figure 7. An illustration of why when two types of carets are involved, the number of carets gets multiplied. The tree on the top left has only horizontal subdivisions, so when joined with a tree with only vertical ones, these have to be put at every leaf.

Lemma 4.1. The word length of an element of $2 V$ that is represented by a tree-pair diagram of depth $D$ is at least $D / 3$.

Proof. Right multiplying by a generator in the finite generating set can add levels to the tree-pair diagram. When we right multiply by a generator, we may need to add carets to $T_{-}$(and thus to $T_{+}$) if the carets in the generator are not already present in $T_{-}$. This can add at most 3 levels, which happens only in the case that we rightmultiply an element $\left(T_{+}, \pi, T_{-}\right)$with $T_{-}$having no right subtree by any of $A_{1}, B_{1}$ or $C_{1}$ from the finite generating set. In that case, the right child of the root of $T_{-}$ is a leaf labeled $n$ and we will need to add carets to it and the corresponding leaf from $T_{+}$to obtain a representative compatible with multiplication by the generator. If leaf $\pi^{-1}(n)$ in $T_{+}$is at maximal level, then the level could increase but only by the number of carets added to leaf $\pi^{-1}(n)$ in $T_{-}$. Thus, any product of less than $D / 3$ generators cannot have depth $D$.

From this it follows that the cyclic subgroups generated by any single generator from the $A_{n}$ or $B_{n}$ families are undistorted in $2 V$, for example.

As seen in the proof of Theorem 3.2, multiplying by a generator can increase the number of carets by a multiplicative factor. The powers of the element $C_{0}$ have exponentially many carets. In fact, the minimal number of carets needed to represent $C_{0}^{n}$ is $2^{n}$. We give further related specific examples later in Section 5. Any of these examples show that the lower bound of Theorem 3.2 is optimal. 
To analyze the genericity of the upper bound in Theorem 3.2, we use arguments analogous to those for $V$. Birget [2004] showed not only that $n \log n$ is an upper bound on the growth of elements in $V$ with respect to the number of carets $n$ in minimal length representatives, but also that the fraction of elements close to this bound converges exponentially fast to 1 . In $2 \mathrm{~V}$, we note analogous behavior:

Theorem 4.2. Let $H_{n}$ be the set of elements of $2 \mathrm{~V}$ representable with $n$ carets and having no representatives with fewer than $n$ carets. The fraction of elements of $H_{n}$ that have word length greater than $n \log n$ converges exponentially fast to 1 .

Proof. Here we use a counting argument, analogous to that used by Birget [2004] to count elements in $V$. In $2 V$, we consider the subset of representatives of elements that have only horizontal subdivisions in the domain and only vertical subdivisions in the range. Their tree-pair diagrams are guaranteed to be reduced and of minimal size by a straightforward argument. So to count the set of elements of this type of diagram size $n$, we have $C_{n}$ choices (where $C_{n}$ is the $n$-th Catalan number) for the first all-square tree of size $n$ and $C_{n}$ choices for the all-triangular tree of size $n$, and $n$ ! choices for the permutation. This set of elements has size $C_{n}^{2} n$ !, which is, by the Stirling formula,

$$
\left(C_{n}\right)^{2} n !=\left(\frac{(2 n) !}{(n+1) ! n !}\right)^{2} n !=\sqrt{\frac{2}{\pi}} \frac{16^{n}}{e^{n}} n^{(n-2)}(1+o(1)),
$$

where the $o(1)$ term goes to zero as $n$ increases.

The number of elements of word length $n$ in any finitely generated group with $d$ generators is no more than $(2 d)^{n}$. Thus we see that the ratio of elements that have word length less than $n \log _{2 d} n$ out of elements that have tree-pair diagrams of size $n$ is less than

$$
\frac{n^{n}}{\sqrt{\frac{2}{\pi}} \frac{16^{n}}{e^{n}} n^{(n-2)}(1+o(1))} \sim c \frac{n^{2} e^{n}}{16^{n}},
$$

which converges to 0 exponentially fast, as desired. So by complementing we have the result.

\section{Distortion}

Elements of $2 \mathrm{~V}$ can be represented with pairs of binary trees, where the carets have been subdivided in two types. This fact makes it more difficult to multiply elements whose caret types disagree, as illustrated already in Theorem 3.2, and the number of carets can grow faster because of this situation. This phenomenon has been described already by Wladis [2007] for the group $F(2,3)$, which has also carets of two types (binary and ternary). This feature of $2 \mathrm{~V}$ implies now that 


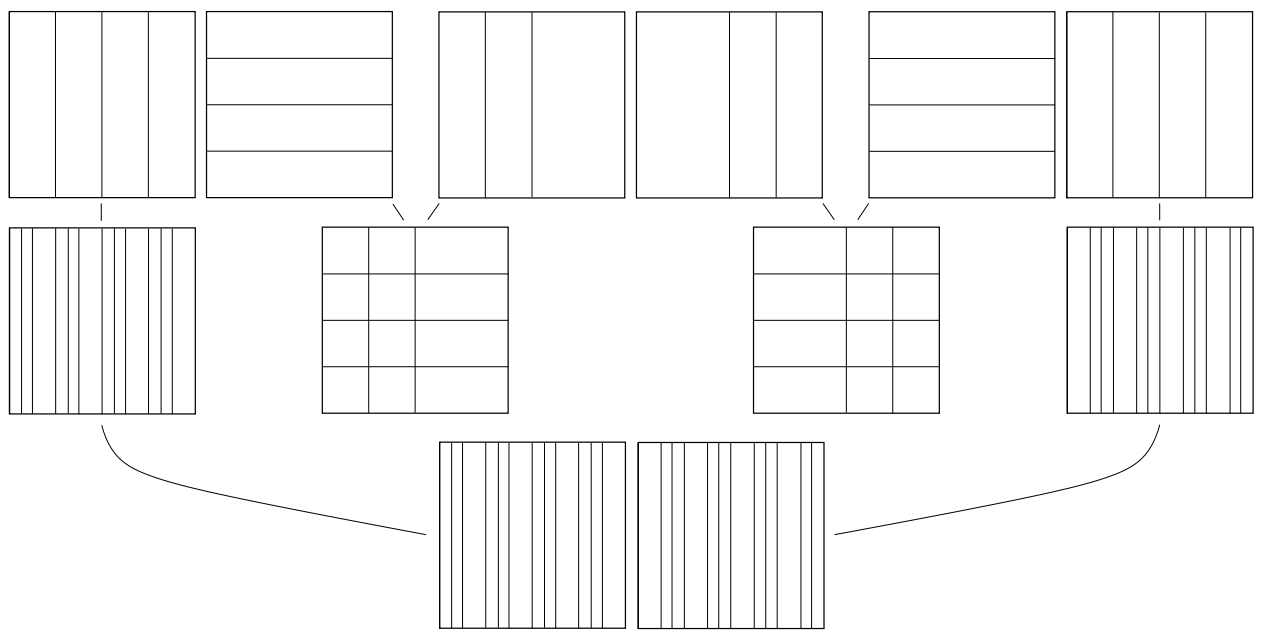

Figure 8. The process of multiplication for $C_{0}^{-2} A_{0} C_{0}^{2}$, illustrating the exponential number of subdivisions.

the groups $F, T$ and $V$, when seen as subgroups of $2 V$ (by doing only vertical subdivisions, for instance), are exponentially distorted.

Theorem 5.1. The groups $F, T$ and $V$ are at least exponentially distorted in $2 V$.

Proof. We consider the specific element $C_{0}^{-n} A_{0} C_{0}^{n}$, illustrated in Figure 8. This element lies in a copy of $F$ in $2 V$ obtained by putting $F$ into $2 V$ using only vertical subdivisions.

The element $C_{0}^{n}$ has $2^{n}$ carets, as seen with an easy induction. Its two trees are balanced trees of depth $n$, one with only vertical subdivisions, and one with only horizontal subdivisions. By matching the horizontal subdivisions with the vertical ones in $A_{0}$, we see the element $C_{0}^{-n} A_{0} C_{0}^{n}$ has a number of carets of order $2^{n}$, and all the carets are of vertical type, so the element is in $F$. This element has length in $V$ no more than $2 n+1$, but the number of carets is exponential in $n$ and thus its word length as an element of the vertical copy of $F$ in $2 V$ is also exponential.

Thus $F$ is at least exponentially distorted in $2 V$. Since $F$ is undistorted in $T$ and $V$, we see that $T$ and $V$ (as subgroups using only vertical subdivisions) are also at least exponentially distorted in $2 \mathrm{~V}$.

\section{Acknowledgments}

The authors are grateful for helpful conversations with Matt Brin, Melanie Stein and Claire Wladis. 


\section{References}

[Birget 2004] J.-C. Birget, "The groups of Richard Thompson and complexity", Internat. J. Algebra Comput. 14:5-6 (2004), 569-626. MR 2006g:20048 Zbl 1088.20016

[Brin 2004] M. G. Brin, "Higher dimensional Thompson groups", Geom. Dedicata 108 (2004), 163-192. MR 2005m:20008 Zbl 1136.20025

[Brin 2005] M. G. Brin, "Presentations of higher dimensional Thompson groups", J. Algebra 284:2 (2005), 520-558. MR 2007e:20062 Zbl 1135.20022

[Burillo et al. 2001] J. Burillo, S. Cleary, and M. Stein, "Metrics and embeddings of generalizations of Thompson's group F”, Trans. Amer. Math. Soc. 353:4 (2001), 1677-1689. MR 2001k:20087 Zbl 0989.20030

[Burillo et al. 2009] J. Burillo, S. Cleary, M. Stein, and J. Taback, "Combinatorial and metric properties of Thompson's group T", Trans. Amer. Math. Soc. 361:2 (2009), 631-652. MR 2010e:20061 Zbl 05518616

[Cannon et al. 1996] J. W. Cannon, W. J. Floyd, and W. R. Parry, "Introductory notes on Richard Thompson's groups”, Enseign. Math. (2) 42:3-4 (1996), 215-256. MR 98g:20058 Zbl 0880.20027

[Wladis 2007] C. Wladis, Metric Properties of Thompson's Groups $F(n)$ and $F(n, m)$, thesis, The City University of New York, 2007.

Received October 21, 2008. Revised October 6, 2009.

JOSÉ BURILLO

DEPARTAMENT DE MATEMÀTICA APLICADA IV

Universitat Politècnica de CATALUnya

Escola Politècnica, SuPERIOR DE CASTELldEFElS

Avda. Del Canal Olímpic 15

08860 CASTELLDEFELS

SPAIN

burillo@mat.upc.es

http://www-ma4.upc.edu/ burillo/

\section{SEAN Cleary}

DEPARTMENT OF MATHEMATICS

The City College of New York

Convent Ave at 138Th St.

NEW YORK, NY 10031

UNITED STATES

cleary@sci.ccny.cuny.edu

http://www.sci.ccny.cuny.edu/ cleary 


\title{
PACIFIC JOURNAL OF MATHEMATICS
}

\author{
http://www.pjmath.org \\ Founded in 1951 by \\ E. F. Beckenbach (1906-1982) and F. Wolf (1904-1989)
}

\section{EDITORS}

V. S. Varadarajan (Managing Editor)

Department of Mathematics

University of California

Los Angeles, CA 90095-1555

pacific@math.ucla.edu

Vyjayanthi Chari

Department of Mathematics

University of California

Riverside, CA 92521-0135

chari@math.ucr.edu

Robert Finn

Department of Mathematics Stanford University

Stanford, CA 94305-2125

finn@math.stanford.edu

Kefeng Liu

Department of Mathematics

University of California

Los Angeles, CA 90095-1555

liu@math.ucla.edu
Darren Long

Department of Mathematics

University of California

Santa Barbara, CA 93106-3080

long@math.ucsb.edu

Jiang-Hua Lu

Department of Mathematics

The University of Hong Kong

Pokfulam Rd., Hong Kong jhlu@maths.hku.hk

Alexander Merkurjev

Department of Mathematics

University of California

Los Angeles, CA 90095-1555

merkurev@math.ucla.edu
Sorin Popa

Department of Mathematics University of California

Los Angeles, CA 90095-1555 popa@math.ucla.edu

Jie Qing

Department of Mathematics

University of California

Santa Cruz, CA 95064

qing@cats.ucsc.edu

Jonathan Rogawski

Department of Mathematics

University of California

Los Angeles, CA 90095-1555

jonr@math.ucla.edu

\section{PRODUCTION}

pacific@math.berkeley.edu

\begin{abstract}
Silvio Levy, Scientific Editor Matthew Cargo, Senior Production Editor
\end{abstract}
ACADEMIA SINICA, TAIPEI

CALIFORNIA INST. OF TECHNOLOGY

INST. DE MATEMÁTICA PURA E APLICADA

KEIO UNIVERSITY

MATH. SCIENCES RESEARCH INSTITUTE

NEW MEXICO STATE UNIV.

OREGON STATE UNIV.

\section{SUPPORTING INSTITUTIONS}

STANFORD UNIVERSITY
UNIV. OF BRITISH COLUMBIA
UNIV. OF CALIFORNIA, BERKELEY
UNIV. OF CALIFORNIA, DAVIS
UNIV. OF CALIFORNIA, LOS ANGELES
UNIV. OF CALIFORNIA, RIVERSIDE
UNIV. OF CALIFORNIA, SAN DIEGO
UNIV. OF CALIF., SANTA BARBARA

UNIV. OF CALIF., SANTA CRUZ

UNIV. OF MONTANA

UNIV. OF OREGON

UNIV. OF SOUTHERN CALIFORNIA

UNIV. OF UTAH

UNIV. OF WASHINGTON

WASHINGTON STATE UNIVERSITY

These supporting institutions contribute to the cost of publication of this Journal, but they are not owners or publishers and have no responsibility for its contents or policies.

See inside back cover or www.pjmath.org for submission instructions.

The subscription price for 2010 is US \$420/year for the electronic version, and \$485/year for print and electronic.

Subscriptions, requests for back issues from the last three years and changes of subscribers address should be sent to Pacific Journal of Mathematics, P.O. Box 4163, Berkeley, CA 94704-0163, U.S.A. Prior back issues are obtainable from Periodicals Service Company, 11 Main Street, Germantown, NY 12526-5635. The Pacific Journal of Mathematics is indexed by Mathematical Reviews, Zentralblatt MATH, PASCAL CNRS Index, Referativnyi Zhurnal, Current Mathematical Publications and the Science Citation Index.

The Pacific Journal of Mathematics (ISSN 0030-8730) at the University of California, c/o Department of Mathematics, 969 Evans Hall, Berkeley, CA 94720-3840, is published monthly except July and August. Periodical rate postage paid at Berkeley, CA 94704, and additional mailing offices. POSTMASTER: send address changes to Pacific Journal of Mathematics, P.O. Box 4163, Berkeley, CA 94704-0163.

PJM peer review and production are managed by EditFLOW ${ }^{\mathrm{TM}}$ from Mathematical Sciences Publishers.

PUBLISHED BY PACIFIC JOURNAL OF MATHEMATICS

at the University of California, Berkeley 94720-3840

A NON-PROFIT CORPORATION

Typeset in LATEX

Copyright $(\mathrm{C} 2010$ by Pacific Journal of Mathematics 


\section{PACIFIC JOURNAL OF MATHEMATICS}

Volume $248 \quad$ No. $1 \quad$ November 2010

An existence theorem of conformal scalar-flat metrics on manifolds with boundary 1

SÉRgio de Moura Almaraz

Parasurface groups

KHALID BOU-RABEE

Expressions for Catalan Kronecker products

Andrew A. H. Brown, Stephanie Van Willigenburg and Mike

ZABROCKI

Metric properties of higher-dimensional Thompson's groups

José BURILLO and SEAN CLEARY

Solitary waves for the Hartree equation with a slowly varying potential

KIRIL DATCHEV and IVAN VENTURA

Uniquely presented finitely generated commutative monoids

PEDRO A. GARCíA-SÁNCHEZ and IgnaCio OJEDA

The unitary dual of $p$-adic $\widetilde{\operatorname{Sp}(2)}$

MARCELA HANZER and IVAN MATIĆ

A Casson-Lin type invariant for links

ERIC HARPER and NIKOLAI SAVELIEV

Semiquandles and flat virtual knots

ALLISON HENRICH and SAM NELSON

Infinitesimal rigidity of polyhedra with vertices in convex position

IVAN IZMESTIEV and JEAN-MARC SCHLENKER

Robust four-manifolds and robust embeddings

VYACHESLAV S. KRUSHKAL

On sections of genus two Lefschetz fibrations

SINEM ÇELIK ONARAN

Biharmonic hypersurfaces in Riemannian manifolds

YE-LIN OU

Singular fibers and 4-dimensional cobordism group 